\title{
A Robotic Microscope for 3D Time-Lapse Imaging Early Stage Salamander Embryos
}

\author{
Susan J. Crawford-Young \\ Department of Electrical and Computer Engineering, University of Manitoba, Winnipeg, Manitoba, R3T 5V6, Canada \\ (mudworks@cici.mb.ca) \\ Richard Gordon \\ Departments of Radiology, Computer Science and Electrical \& Computer Engineering \\ University of Manitoba, Winnipeg, Manitoba,R3A1R9, Canada (gordonr@cc.umanitoba.ca)
}

\begin{abstract}
A robotic microscope was designed using a microcontroller with three stepper motors to control three-axis movement. Two 7 megapixel digital cameras controlled by the microcontroller capture images when the stage moves into position. Using 4 prisms, throughfocus time-lapse digital pictures of six views of Ambystoma mexicanum embryos (axolotl, a salamander) are taken every 5 minutes for 52 hours of early development, from fertilization to stage 20, i.e., neural tube closure. In-focus views [Gord83] of all sides of the embryo are calculated using several image fusion techniques. In the early embryo surface epithelial cells differentiate to form neural tissue and external skin tissue. Observing the whole embryo surface at cellular level will give a better idea of the stress and strain each cell undergoes and physical forces involved in cell differentiation, including waves of cell surface expansion or contraction Time-lapse photographs capture the dynamics of the embryo during development, that is lost in histological techniques that use non-living material.
\end{abstract}

\section{Introduction}

A robotic microscope has been built for the purpose of viewing living $2 \mathrm{~mm}$ opaque

spherical axolotl salamander (Ambystoma mexicanum) embryos from all sides. Axolotl embryos are bottom heavy and much of the developmental dynamics takes place on the exterior surface. They start as eggs laid in water and grow using internal yolk for energy until hatching in 2-3 weeks. The embryos stay round at $2 \mathrm{~mm}$ diameter up to neural tube closure [Bord89]. Digital pictures are taken from six views to see the whole surface of the embryo at cellular resolution, allowing cell

tracking over the surface during this period. Images in our 3D time-lapse database will permit many enquires into the behavior of embryonic cells.

As embryos develop, significant cell movement occurs, causing a variety of stresses on the embryo surface that may have a significant role to play in cell differentiation [Gord85; Gord99; Belo06, Ingb06]. Physical force on cells is transmitted to the nucleus and causes changes in gene expression which in turn changes cell type [Berf97; Ben-91; Janm98; Helm03].

The study of embryo development, using time-lapse photography, dates back to 1929 cinematography of rabbit eggs [Lewi29]. Embryos develop slowly, somewhat like plant growth. Time-lapse imaging makes developmental movements apparent. To capture the movement of cells Hara made time-lapse recordings of the top and bottom of an axolotl egg starting at a single cell with frames taken every 30 seconds. At 24 frames per second viewing (x720) he saw the motion of waves of cell stiffening on the surface during cell division [Hara71]. Most embryological observation uses serial sectioning of dead processed tissue, causing dynamic details to be missed [Dist06; Wein04].

Most modern embryology is concerned with genetics and gene products and is mainly descriptive [Roel05]. There is a wide range of genetic research that has been done on embryos using florescent molecules to mark proteins and other gene products. These techniques do not explain the physical behavior of cells in embryos or the forces they generate and respond to. Using descriptions of what is happening is only part of the puzzle of embryology. It is like trying to tell how a car works by describing its parts [Roel05]. 
Viewing the surface of the embryo in early development will allow us to closely monitor the process of early cell differentiation. Ectodermal cells change into nerve cell and skin cell precursors on the surface of the embryo. This is one of the first types of cell differentiation to occur in an embryo and is the beginning of the brain and spinal cord. The forces exerted on the cells can be studied by tracking cell shape and cell movements in relation to one another.

One of the major cell behaviors is cell contraction, which has been observed to occur in waves on the surface of the embryo [Gord98; Gord94; Nieu99; Brod94; Gord99; Gord96]. These waves need to be tracked to see if they correlate with nerve cell development, calcium ion gradients and protein expression. Cell position, size and shape versus time will give a map of the strain field on the surface of the embryo.

\section{Apparatus}

We designed a robotic microscope based on a microcontroller with built in stepper motor drivers to move an $\mathrm{X}, \mathrm{Y}, \mathrm{Z}$ axis stage and operate two cameras attached to two microscopes to take time-lapse pictures of a whole embryo. The microcontroller stepper motor drivers have a separate chip for each axis, and with 1/16 micro-stepping capability create accurate and repeatable movements of each axis. The top microscope has tight physical tolerances as it has a $1 \mathrm{~mm}$ working distance. The robotic movement has to work within this range to acquire $\mathrm{Z}$ stack images for extended depth of field imaging by moving $0.33 \mathrm{~mm} \pm 0.01 \mathrm{~mm}$ over $1 \mathrm{~mm}$. The mechanical and electrical stops are within several micrometers of each other and the movement of the $\mathrm{Z}$ axis is sandwiched between the two microscopes, making precision design a necessity.

The apparatus includes two microscopes, a motorized microscope stage $(\mathrm{X}, \mathrm{Y}, \mathrm{Z})$, four prisms, a microcontroller, two ring lights, two digital cameras, and a PC computer with a 2 Gigabyte memory, 250 GB harddrive and $250 \mathrm{~GB}$ of external digital storage. The system is a standard microcontroller based set up that is easy to program from a pull down menu.

The sides of the embryo are viewed through four right angle prisms, which project 4 side images downward to the bottom microscope. The X,Y stepper motors for the stage move the embryo into position and then the $\mathrm{Z}$ axis stepper motor moves it up and down to get three in-focus views for each side of the embryo and then three views of the bottom of the embryo. The $\mathrm{Z}$ axis then moves up in range of the top microscope to get three more throughfocus views. The motors are controlled by a Simplestep microcontroller (xyzmicro). The microcontroller operates the lights and cameras after moving the stage into position. The movement is repeated for each side of the embryo to obtain views of all sides of the embryo every five minutes for 52 to 72 hours. Communications to the Simplestep board is via a USB2 to Keyspan serial adapter. The microcontroller was run from the computer but does have the capability of programming an on board EPROM for stand-alone operation.

The motors were programmed (in the Simplestep sswin program) to move to home position, which is the home sensor on the $\mathrm{X}, \mathrm{Y}$ stage and the bottom Hall sensor on the $\mathrm{Z}$ axis, then moved into the 5 bottom positions. The $\mathrm{Z}$ axis is moved to take three through-focus images of each position with a 15 second delay between each to give the camera time to take a picture in the low light conditions inside the prism holder. There was a one second delay needed between the ON and OFF of the outputs to the cameras to ensure operation of the relays and remote camera controls attached to them. The $\mathrm{Z}$ axis is then moved to home and then up to the limits of the upper Hall sensor and mechanical stops to get three throughfocus pictures with the top microscope and camera. The movement of the $\mathrm{Z}$ axis linear drive stepper motor is approximately 4500 steps for $1 \mathrm{~cm}$ of movement. A Danaher Precision Systems kv Ultra compact positioner was used for the $\mathrm{Z}$ axis. The $\mathrm{X}, \mathrm{Y}$ axes were made from a used Zeiss $X, Y$ stage with the gear box removed and two NEMA 14 bipolar stepper motors replacing the original high resistance steppers. The Hall sensors on the $\mathrm{Z}$ axis were powered separately with a voltage regulator as they needed more current than available on the Simplestep board.

The Simplestep microcontroller worked well for this application, the speed and power parameters used were on the low side and nowhere near the upper capabilities of the board. Since the microcontroller comes with built in stepper motor drivers that do precision microstepping for under $\$ 500$ US designing a new system of this sort would be redundant. The challenge with the apparatus was in finding appropriate motors, sensors and other peripherals that would work in a trouble free fashion.

The cameras for the project needed to be digital with at least a 5 mega pixel square sensor, with 8 micrometer pixel size, and one inch diameter chip size to get the resolution needed for imaging $10 \square \mathrm{m}$ embryo cells on a $2 \mathrm{~mm}$ embryo with a $4 \mathrm{x}$ microscope objective lens. The cameras have to load images directly into the computer and need to have live view for set up. We chose two 7.4 megapixel E330 Olympus cameras. The camera system design included a modified battery for the cameras so that the cameras could operate continuously without running out of battery power. The camera remote 
controls were battery operated without wires. They were modified by placing wires on both sides of the on push button so that they could be operated from relays hooked to two outputs of the microcontroller. The 7.4 $\square \mathrm{m}$ CMOS chips for the camera are theoretically capable of $15 \square \mathrm{m}$ resolution. When the image is enlarged with a $4 x$ microscope objective $15 / 4$ gives a resolution of the enlarged object at approximately $4 \square \mathrm{m}$. Taking into account Nyquest's theorem the resolution is $8 \square \mathrm{m}$, which is the resolution we were seeking. Cellular resolution was achieved because we saw the characteristic polygonal shapes of cells in tissues [Bray92].

The microscopes are a Wild M8 (TYP376788) stereomicroscope with camera mount, inverted for the bottom views of the embryo. The top microscope is a homemade microscope using a Nikon 4/0.13WI (160 $\mathrm{mm}$ focal length) water immersion $4 \mathrm{X}$ lens. The bottom microscope sees the bottom of the embryo and the four sides of the embryo through small $3 \mathrm{~mm}$ prisms placed in an embryo holder. The prisms are TECH SPEC ${ }^{\mathrm{TM}}$ HighTolerance Right Angle Prisms.

A holder for the $2 \mathrm{~mm}$ embryos was designed using four prisms and modified Petri dishes. The For a clearer view of the embryo from the bottom a $19 \mathrm{~mm}$ hole was cut in the centre of the dish and a $22 \mathrm{~mm}$ glass microscope cover slip was added. The glass cover slip, a Fisher \#12541B $22 \times 22 \mathrm{~mm}$ and was glued to the edges of the hole in the centre of the Petri dish. The gluing procedure used melted wax, which was heated by a heat gun and ran under the cover slip sealing it to the Petri dish. Bees wax was used as it is a more natural substance and less likely to harm the axolotl embryos. Its use in embryology is standard [Hamb60].

The embryo holder fit over top of the cover slip and was held using Fun-Tack for a removable, nontoxic glue down. All parts of the holder were washed with $70 \%$ isopropyl alcohol prior to using on live eggs.

The prisms were held in place with a machined piece of acrylic plastic. The clamps for the prisms were made by drilling and taping set screws into the acrylic plastic on the four sides of the holder. When the screws are turned the acrylic plastic bends slightly clamping the prisms in place. The prisms were then covered with a protective layer of bees wax, to keep their baking from being exposed to the saline Holtfreter's solution and for mechanical protection. A hole was drilled towards on of the prisms to provide an entry for a thermistor wire to measure temperature. Five views of the embryos can be seen at the same time from the bottom microscope but for better resolution each prism and the centre area were photographed separately.
The eggs used for viewing were white axolotl eggs because they have natural pigment spots that will make cell tracking easier to do. The wild type eggs also have pigment markings but they are more crowded and the albino eggs are hard to view because they are completely white so details on the surface are hard to see.

The eggs were partially de-jelled so that they would fit into the embryo holder and so that exterior dirt was removed from the gel. The diameter of the egg and jell needs to be approximately $3.5 \mathrm{~mm}$ to fit into the embryo holder. Some of the embryos gel is kept to place on top of the embryo once it is in the holder to make a more even light path of jell for the top water immersion microscope objective.

The egg was placed in the embryo holder and then covered with $25 \%$ Holtfreter's solution in de-ionized water [Hamb60]. Holtfreter's is a weak salt solution developed for working with amphibian embryos and is necessary because dejelled eggs burst in plain de-ionized water. The final configuration for the embryo holder includes an additional flexible extension so that water solution can be filled to a depth so that the top microscope, which has a water immersion objective, does not leave the water during the time-lapse photography. This is to prevent bubbles from forming on this lens.

\section{Image Processing}

The images from the microscope were on the dark side due to the prisms blocking the light. Special fiberoptic holders were made to direct the light from four bright white LEDs into the prism holder and epi-illumination was used by placing four bright white LEDs in the left optic of the bottom stereo-microscope.

The dark images were then flat fielded using image division in with ImageJ software. Then the images were brightened using adaptive histogram equalization. The in focus views of each time-position were then registered and fused with ImageJ software using wavelet techniques [Fors04]. The real wavelet and complex wavelet methods were found to give similar results using image subtraction to determine their similarity. The faster real wavelet methods of image fusion can therefore be used on the images without much degradation. 


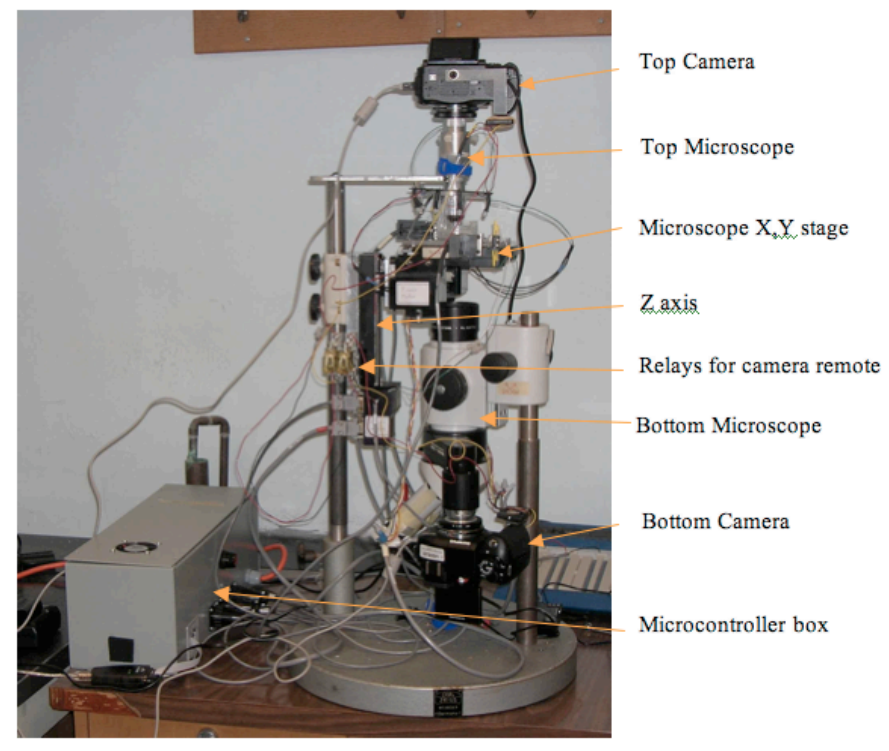

Figure 1 Microscope setup and control

\section{Conclusion}

We have made a microscope that can view a developing embryo from all sides and take time-lapse in-focus images. This will allow us to study developmental dynamics and determine the stresses that occur on the surface of a $2 \mathrm{~mm}$ embryo during development. The microscope could be used for the study of other $2 \mathrm{~mm}$ size embryos such as spruce embryos or to study flocculation and crystal formation.

Physical forces acting on cells do cause changes in their cytoskeleton, extra-cellular matrix and gene expression [Janm98; Ingb06]. Study of many classical embryological experiments in which embryos are poked, cut and/or have tissue transplantation done to them should be revisited with this insight.

Our knowledge of embryology will not be complete until the forces on cells in a developing embryo can be evaluated and correlated with the molecular biology that gives rise to changes in gene expression.

\section{Acknowledgements}

Supported in part funds from the Manitoba Institute for Child Health and the Canadian Space Agency.

\section{References}

[Belo06] Beloussov, L. V., Luchinskaya, N. N., Ermakov, A. S., and
Glagoleva, N. S., "Gastrulation in amphibian embryos, regarded as a succession of biomechanical feedback events", Int. J. Dev. Biol.,50: 113-122, 2006

[Ben-91] Avri Ben-Ze'ev, "Animal Cell Shape Changes and Gene Expression”, BioEssays 13(5) May 1991

[Berf97] Anne K. Berfield, Douglas Spicer and Christine K. Abrass, "Insulin-like Growth Factor I (IGF-I) Induces Unique Effects in the Cytoskeleton of Cultured Rat Glomerular Mesangial Cells", The Journal of Histochemistry \& Cytochemistry, 45(4): 583-593, 1997

[Brod94] G.W. Brodland, R. Gordon, M.J. Scott, N.K. Björklund, K.B. Luchka, C.C. Martin, C. Matuga, M. Globus, S. Vethamany-Globus \& D. Shu, "Furrowing surface contraction wave coincident with primary neural induction in amphibian embryos." J. Morphol. 219(2): 131-142, 1994

[Bord89] N.P. Bordzilovskaya, T.A. Dettlaff, S.T. Duhon and G.M. Malacinski, Developmental-stage series of axolotl embryos. In: Armstrong, J.B. \& G.M. Malacinski, "Developmental Biology of the Axolotl”, New York: Oxford University Press, p. 201-219, 1989

[Bray92] Dennis Bray, "Cell movements", Garland Publishing Inc., page 55,1992

[Dist06] Martin Distel, Andreas Babaryka, and Reinhard W. Köster, "Multicolor In Vivo Time-Lapse Imaging at Cellular Resolution by Stereomicroscopy", Developmental Dynamics, 235:1100-1106, 2006

[Fors04] B. Forster, D. Van De Ville, J. Berent, D. Sage, and M. Unser, "Complex Wavelets for Extended Depth-of Field: A New Method for the Fusion of Multichannel Microscopy Images", Microscopy Research and technique, 65: 33-42, 2004

[Gord85] R. Gordon, "A review of the theories of vertebrate neurulation and their relationship to the mechanics of neural tube birth defects”, J. Embryol. Exp. Morph. 89: (Supp1.), 229-255, 1985

[Gord96] R. Gordon, N.K. Björkland, "How to observe surface contraction waves on axolotl embryos", Int J Dev Biol, 40: 913-914, Aug. 1996

[Gord99] R. Gordon, "The Hierarchical Genome and Differentiation Waves: Novel Unification of Development, Genetics and Evolution", Singapore \& London: World Scientific \& Imperial College Press, 1999

[Hamb60] V. Hamburger, "A Manual of Experimental Embryology", Chicago: University of Chicago Press, 1960.

[Hara71] K. Hara, "Cinematographic Observation of "Surface Contraction Waves" (SCW) during the Early Cleavage of Axolotl Eggs", Wilhelm Roux' Archiv (Springer-Verlag), 167: 183--186, 1971

[Helm03] B.P. Helmke, A.B. Rosen, P.F. Davies, "Mapping Mechanical Strain of an Endogenous cytoskeletal Network in Living Endothelial Cells", Biophysical Journal, 84: 2691-2699, 2003

[Ingb06] D. Ingber, "Cellular mechanotransduction: putting all the pieces together again", The FASEB Journal., 20:811-827, 2006

[Janm98] Paul A. Janmey, "The Cytoskeleton and Cell Signaling: component Localization and Mechanical Coupling", Physiological Reviews, 78(3), July 1998

[Lewi29] W.H. Lewis and P.W. Gregory, "Cinematographs of Living Developing Rabbit-Eggs, Science 69: 226-229, 1929 
[Nieu99] P.D. Nieuwkoop, "The neural induction process; its morphogenetic aspects”, Int. J. Dev. Biol., 43: 615-623, 1999

[Roel05] Roeland M.H. Merks, James A. Glazier, "A cell-centered approach to developmental biology”, Physica A, 352: 113-130, 2005

[Weni04] W.J. Weninger, O. Tassy, S. Darras, SH. Geyer, and D.

Thieffry, "From Experimental imaging Techniques to Virtual

Embryology", Hist Phil. Life Sci., 26:355-375, 2004 
